\title{
Decreased Interfacial Tension of Demixed Aqueous Polymer Solutions due to Charge
}

\author{
Mark Vis, ${ }^{1, *}$ Vincent F. D. Peters, ${ }^{1}$ Edgar M. Blokhuis, ${ }^{2}$ Henk N. W. Lekkerkerker, ${ }^{1}$ \\ Ben H. Erné, ${ }^{1, \dagger}$ and R. Hans Tromp ${ }^{1,3}$ \\ ${ }^{1}$ Van 't Hoff Laboratory for Physical and Colloid Chemistry, Debye Institute for Nanomaterials Science, \\ Utrecht University, Padualaan 8, $3584 \mathrm{CH}$ Utrecht, The Netherlands \\ ${ }^{2}$ Colloid and Interface Science, Gorlaeus Laboratories, Leiden Institute of Chemistry, \\ P.O. Box 9502, 2300 RA Leiden, The Netherlands \\ ${ }^{3}$ NIZO food research, Kernhemseweg 2, 6718 ZB Ede, The Netherlands \\ (Received 7 April 2015; revised manuscript received 2 June 2015; published 14 August 2015)
}

\begin{abstract}
Electric charge at the water-water interface of demixed solutions of neutral polymer and polyelectrolyte decreases the already ultralow interfacial tension. This is demonstrated in experiments on aqueous mixtures of dextran (neutral) and nongelling fish gelatin (charged). Upon phase separation, electric charge and a potential difference develop spontaneously at the interface, decreasing the interfacial tension purely electrostatically in a way that can be accounted for quantitatively by Poisson-Boltzmann theory. Interfacial tension is a key property when it comes to manipulating the water-water interface, for instance to create novel water-in-water emulsions.
\end{abstract}

DOI: 10.1103/PhysRevLett.115.078303

PACS numbers: 82.35.Rs, 05.70.Np, 68.05.-n, 83.80.Rs

Coexisting polymeric phases in aqueous solution are extensively studied both in theory and experiment [1-14]. They have important applications in food and biomedicine, as they provide the means to texturize water and to encapsulate a first aqueous phase in a second one, both typically containing $90 \%$ of water [15-22]. Often, one or both polymer species are charged, tunable by varying the $\mathrm{pH}$. The liquid-liquid interface of such systems is quite peculiar, as it is permeable to solvent and small ions, and it has an ultralow interfacial tension, typically in the range of $0.01-10 \mu \mathrm{N} / \mathrm{m}[3,23-33]$. The interplay between the phase separation of charged polymers and a permeable interface leads to an electrical potential difference between the two phases, which, as we show here, further reduces the already ultralow interfacial tension.

An important consequence of the reduction in interfacial tension is that it may become so low that it becomes entropically favorable for the system to increase its interfacial area and to form microdomain structures, as in microemulsions and block copolymer systems. The conditions under which such structures would be present in polyelectrolyte systems were theoretically investigated in Refs. [34-36], but it is fair to say that any clean experimental observation is still lacking. To understand better the influence of charge on the interfacial tension, we therefore experimentally investigate a phase-separated aqueous solution of a neutral polymer and a polyelectrolyte. A deeper understanding of the structure and tension between such polymeric phases would help to pave the way to show experimentally the existence of microstructured polyelectrolyte systems and the formulation of water-in-water emulsions, which mainly remains a matter of empirical research.

When one of the polymer species is charged, the liquidliquid interface is also charged as a consequence of the
Donnan effect: the unequal partitioning of ions over the two phases results in an electrical potential difference, referred to as the Donnan potential [37-39]. Since the Donnan effect not only affects the interfacial tension itself but also the overall phase diagram, it is necessary to determine not only interfacial tension and Donnan potential, but also the precise composition of the coexisting phases. This allows us to examine the effect of interfacial charge on the interfacial tension at equal difference in polymer concentrations between the two phases. We will show that the effect of charge on the interfacial tension can be well understood in terms of Poisson-Boltzmann theory adapted to describe the liquid-liquid interface.

The experiments were performed on phase separating aqueous solutions of dextran $(100 \mathrm{kDa})$ and nongelling fish gelatin $(100 \mathrm{kDa})$ using equal concentrations of both polymers. Dextran is a neutral polymer and gelatin is a weak polyelectrolyte with a charge adjustable via the $\mathrm{pH}$. This model system has been featured in several earlier studies [10,11,29-31,40-42]. The compositions of the phases, the difference in electrical potential, the salt concentration, and the charge on the gelatin were determined as described previously $[40,41]$.

The interfacial tension is experimentally determined from an analysis of the static profile of the liquid-liquid interface at the flat wall of a polystyrene cuvette, see Fig. 1, as done previously for colloid-polymer mixtures [43]. The capillary length $\ell_{\mathrm{c}} \equiv \sqrt{\gamma /(\Delta \rho g)}$ sets the length scale of this profile, where $\gamma$ is the interfacial tension, $\Delta \rho$ is the mass density difference between the two phases, and $g$ is the gravitational acceleration. The profile was imaged using an optical microscope and the resulting value of $\ell_{\mathrm{c}}$ together with a precise determination of $\Delta \rho$ allows us to determine the interfacial tension. 


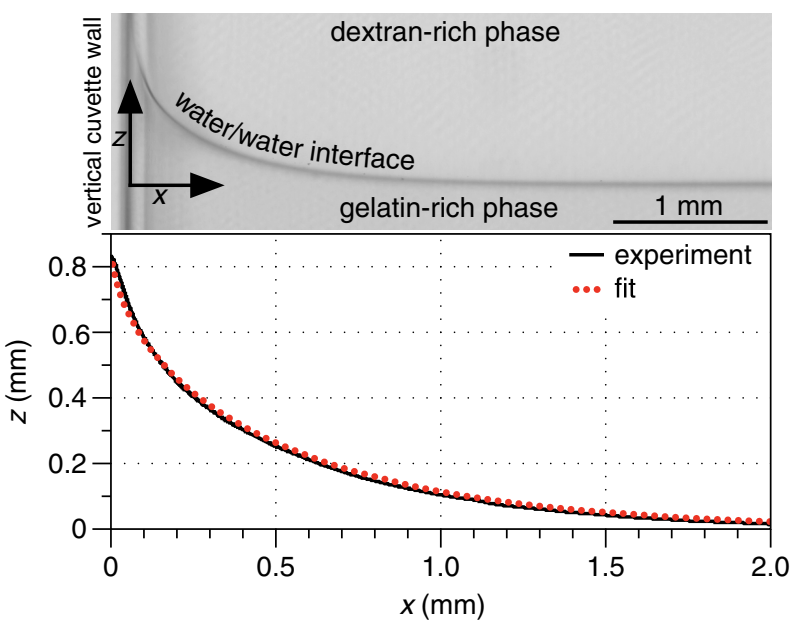

FIG. 1 (color online). Determination of the interfacial tension of a demixed aqueous solution of 5\% dextran and 5\% nongelling fish gelatin from image analysis of the profile of the liquid-liquid interface at a vertical polystyrene surface: (top) micrograph and (bottom) extracted profile and fit.

The electrical potential difference across the interface was varied by changing the charge on the polyelectrolyte. Our fish gelatin has a net zero charge at $\mathrm{pH} 7.5$, is positively charged at low $\mathrm{pH}$, and is negatively charged at higher $\mathrm{pH}$ [40]. In the linear regime $\left(\left|\psi_{D}\right| \leq 10 \mathrm{mV}\right)$, the Donnan potential $\psi_{D}=\psi_{\alpha}-\psi_{\beta}$ is given by $[40,41]$

$$
\psi_{D} \simeq \frac{k T}{e} \frac{z \Delta c_{p}}{2 c_{s}}
$$

where $k T$ is the thermal energy, $e$ is the elementary charge, $z$ is the average net number of charges per polyelectrolyte molecule, $\Delta c_{p}=c_{p, \alpha}-c_{p, \beta}$ is the polyelectrolyte concentration difference between the two phases $\alpha$ and $\beta$, and $c_{s}$ is the concentration of background salt. The two extremes in magnitude of the Donnan potential were obtained by varying the $\mathrm{pH}$ from $\mathrm{pH} 6.2$ with $5 \mathrm{mM}$ salt $(z \approx+5)$, where the Donnan potential was less than $1 \mathrm{mV}$, to $\mathrm{pH} 4.8$ with $9 \mathrm{mM}$ salt $(z \approx+20)$, where we measured the highest Donnan potentials, for instance $\psi_{D}=7.26 \pm 0.04 \mathrm{mV}$ at $7.5 \%$ by mass of each polymer. In these two cases, the position of the binodal is quite different in the phase diagram of demixing, see Fig. 2, and the critical demixing mass fraction shifts by a factor of 2 . Such a shift has been observed also in similar systems [44,45] and can be understood in terms of the loss in translational entropy of the salt ions [41]. The polyelectrolyte-rich phase has the highest concentration of counterions, which stems from the condition of macroscopic electroneutrality, and this unequal distribution of the ions over the two phases has an entropic cost. The result is that it is more difficult to achieve phase separation as the charge on the polyelectrolyte increases.

From Fig. 2, it is clear that to understand the direct effect of charge on the interfacial tension at different Donnan potentials, one cannot compare the interfacial tensions at

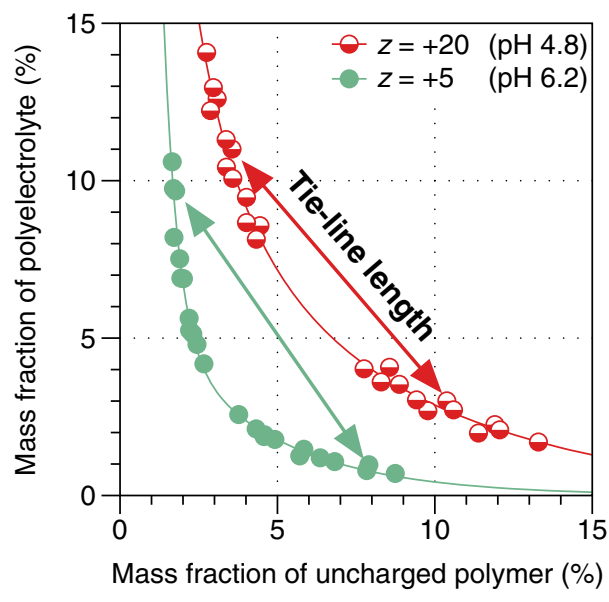

FIG. 2 (color online). Measured phase diagrams [40,41] of demixing of aqueous solutions of dextran (uncharged) and nongelling fish gelatin (charged), under conditions where the Donnan potential is small ( $\mathrm{pH} 6.2,5 \mathrm{mM}$ salt) and where it is large (pH 4.8 with $9 \mathrm{mM}$ salt, 20 charges per gelatin chain).

equal overall concentration of the polymers: in some cases where phase separation does occur at relatively low charge on the polyelectrolyte, no demixing occurs at higher charge. To account for the shift in the binodal due to charge, we need to introduce some measure for the position in the phase diagram. Several choices are possible such as the difference in polymer concentration or difference in polyelectrolyte concentration, but here we have taken the tie-line length as a measure for the position in the phase diagram. It is defined as the distance between the coexisting points on the binodal at particular overall polymer concentrations, polyelectrolyte charge, and salt concentration (see Fig. 2). To investigate the scaling behavior of the interfacial tension or the effect of charge on the interfacial tension, the particular choice to denote the position in the phase diagram has little consequence.

The measured interfacial tensions as a function of tie-line length are shown in Fig. 3 for the two pH values of Fig. 2 and for $\mathrm{pH} 9.2$ with $7 \mathrm{mM}$ salt, where the number of charges on the polyelectrolyte $(z \approx-6)$ is similar in magnitude but opposite in sign compared to the situation at $\mathrm{pH}$ 6.2. A first observation is that the interfacial tension scales with the tieline length to the power 3.3. This is somewhat below the theoretically expected exponent of $\mu / \beta=3.8$, with $\mu=1.26$ and $\beta=0.325$ [46], the critical exponent of the interfacial tension and concentration difference, respectively. A second observation is that - at equal tie-line length — the interfacial

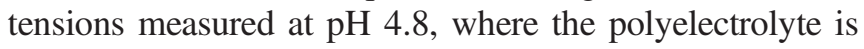
relatively highly charged, are significantly lower than those at $\mathrm{pH} 6.2$ and $\mathrm{pH}$ 9.2, where the Donnan potential is lower. To come to a quantitative description of this effect, we calculate the electrostatic contribution to the interfacial tension using Poisson-Boltzmann theory.

It is convenient to model our phase-separated liquid-liquid system as the sum of two electrical double layers. To show this, we first consider the effect of interfacial charge on a 


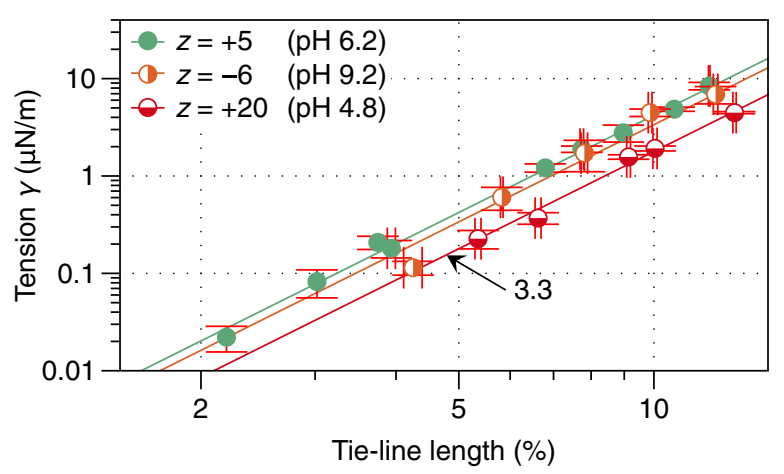

FIG. 3 (color online). Interfacial tension between demixed aqueous solutions of dextran and nongelling fish gelatin. The experimentally measured tension is plotted against the tie-line length (Fig. 2) at increasing absolute number of charges and Donnan potential: $\mathrm{pH} 6.2$ with $5 \mathrm{mM}$ salt, $\mathrm{pH} 9.2$ with $7 \mathrm{mM}$ salt, and $\mathrm{pH} 4.8$ with $9 \mathrm{mM}$ salt.

single electrical double layer. The contribution to the interfacial tension of a single electrical double layer can be calculated with equal results in different ways, for instance via the free energy route or pressure route [47]. Here, we opt for an electrostatics approach resembling that of Frenkel [48], and Verwey and Overbeek [49] for a charged solid-liquid interface; similar approaches have been used to describe charged oil-water and air-water interfaces [50-52]. The surface free energy is then calculated by integrating the potential-dependent surface charge density $\sigma(\Psi)$ over the dimensionless potential $\Psi \equiv \psi e /(k T)$, with $\psi$ the electrical potential $[48,49]$ :

$$
\frac{F}{A} \equiv f=-\frac{k T}{e} \int_{0}^{\Psi_{0}} d \Psi \sigma(\Psi),
$$

where $\Psi_{0}$ is the potential at the solid substrate. An expression for $\sigma(\Psi)$ is obtained starting from the Poisson-Boltzmann equation,

$$
\frac{d^{2} \Psi}{d Z^{2}}=\sinh (\Psi)
$$

where $\Psi(Z)$ is the electrical potential at a dimensionless distance $Z \equiv \kappa z$ from the interface, and $\kappa$ is the Debye screening constant, given by $\kappa \equiv \sqrt{8 \pi \lambda_{B} c_{s}}$. Here, the Bjerrum length is given by $\lambda_{B} \equiv e^{2} /\left(4 \pi \epsilon \epsilon_{0} k T\right)$, where $\epsilon_{0}$ is the permittivity of vacuum and $\epsilon$ is the relative permittivity of the solvent (water). Integration of Eq. (3) yields

$$
\frac{d \Psi}{d Z}=-2 \sinh \left(\frac{\Psi}{2}\right)
$$

where we used that $\Psi$ and $d \Psi / d Z$ are zero far from the substrate. Using electroneutrality of the system, Eq. (4) leads to the following surface charge density for a surface potential $\Psi_{0}$ :

$$
\sigma=-\frac{e \kappa}{4 \pi \lambda_{B}}\left(\frac{d \Psi}{d Z}\right)_{\Psi=\Psi_{0}}=\frac{e \kappa}{2 \pi \lambda_{B}} \sinh \left(\frac{\Psi_{0}}{2}\right) .
$$

This expression is derived for a single double layer that results from the presence of charge on the solid substrate. In the present system, the interface can be viewed as the sum of two electrical double layers with surface charge densities $\sigma_{\alpha}$ and $\sigma_{\beta}$, joined at a central plane that delimits two parts of equal absolute charge but opposite sign $\left(\sigma_{\alpha}+\sigma_{\beta}=0\right)$. In analogy to Eq. (5), the total electrical potential drop, $\Psi_{D}=\Psi_{\alpha}-\Psi_{\beta}$, is distributed over the two phases, giving for the surface charge densities

$$
\sigma_{\alpha}=\frac{-e \kappa_{\alpha}}{2 \pi \lambda_{B, \alpha}} \sinh \left(\frac{\Psi_{\alpha}}{2}\right), \quad \sigma_{\beta}=\frac{-e \kappa_{\beta}}{2 \pi \lambda_{B, \beta}} \sinh \left(\frac{\Psi_{\beta}}{2}\right),
$$

where we have set the electrical potential zero at the central plane, for convenience, and where we have taken into account that the Debye lengths and Bjerrum lengths in the two phases may be different due to the unequal partitioning of salt and a possible difference in permittivity. It is convenient to define an effective salt concentration $c_{s} \equiv$ $\sqrt{c_{s, \alpha} c_{s, \beta}}$ and relative permittivity $\epsilon \equiv \sqrt{\epsilon_{\alpha} \epsilon_{\beta}}$ so that global values of the Bjerrum and Debye lengths are given by $\lambda_{B} \kappa^{-1}=\sqrt{\omega} \lambda_{B, \alpha} \kappa_{\alpha}^{-1}=\frac{1}{\sqrt{\omega}} \lambda_{B, \beta} \kappa_{\beta}^{-1}$, with $\omega \equiv \sqrt{c_{s, \alpha} \epsilon_{\alpha} /\left(c_{s, \beta} \epsilon_{\beta}\right)}$.

Integration of Eq. (6) as in Eq. (2) results in the following surface free energies of the two double layers:

$$
\begin{aligned}
& f_{\alpha}=-\frac{k T \kappa}{\pi \lambda_{B}} \sqrt{\omega}\left[\cosh \left(\frac{\Psi_{\alpha}}{2}\right)-1\right], \\
& f_{\beta}=-\frac{k T \kappa}{\pi \lambda_{B}} \frac{1}{\sqrt{\omega}}\left[\cosh \left(\frac{\Psi_{\beta}}{2}\right)-1\right] .
\end{aligned}
$$

The electric contribution to the interfacial tension is now simply the sum of these two expressions, $\Delta \gamma=f_{\alpha}+f_{\beta}$.

In order to simplify these expressions further, we may assume that the dimensionless electrical potential is small, $\Psi_{D} \ll 1$. From the electroneutrality condition, $\sigma_{\alpha}+\sigma_{\beta}=0$, it then follows that the individual bulk electrical potentials are approximately given by $\Psi_{\alpha} \simeq$ $\Psi_{D}(1 / 1+\omega)$ and $\Psi_{\beta} \simeq-\Psi_{D}(\omega / 1+\omega)$. Furthermore, the hyperbolic functions in Eqs. (7) and (8) can be expanded to second order. The total electric contribution to the interfacial tension is then

$$
\Delta \gamma \simeq-\left(\frac{\Psi_{D}}{2}\right)^{2} \frac{\sqrt{\omega}}{1+\omega} \frac{k T \kappa}{2 \pi \lambda_{B}} .
$$

Finally, when we also assume that the relative permittivity is the same in both phases, we have that $\omega=$ $\exp \left(-\Psi_{D} / 2\right) \simeq 1$ so that the factor $\sqrt{\omega} /(1+\omega)$ is close to $1 / 2$. The electric contribution to the interfacial tension is then in good approximation given by the following simple expression:

$$
\Delta \gamma \simeq-\frac{k T \kappa}{16 \pi \lambda_{B}} \Psi_{D}^{2} .
$$

This final result is plotted in Fig. 4, together with the unapproximated form obtained by summing Eqs. (7) and (8). 


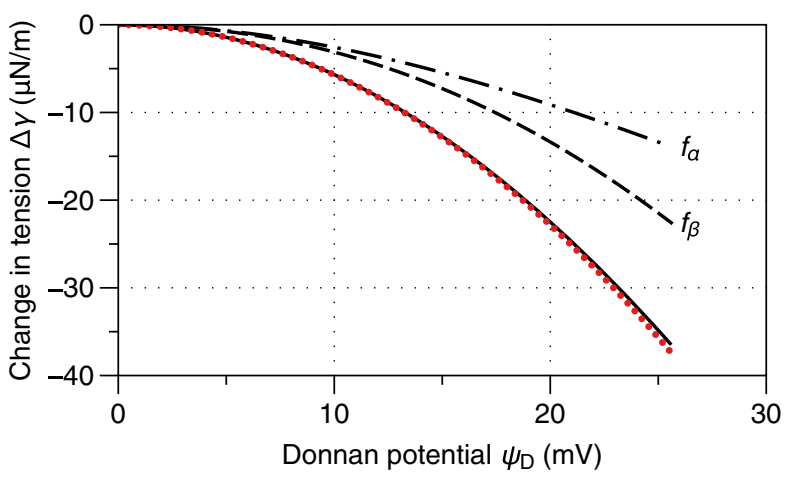

FIG. 4 (color online). Electric contribution to the interfacial tension as a function of the Donnan potential $\psi_{D}$. The contributions $f_{\alpha}$ and $f_{\beta}$ from Eqs. (7) and (8), and their sum $\Delta \gamma=$ $f_{\alpha}+f_{\beta}$ (solid line) are given together with the approximation of Eq. (10) (dotted line). We have taken $c_{s}=\sqrt{c_{s, \alpha} c_{s, \beta}}=10 \mathrm{mM}$, $\epsilon_{\alpha}=\epsilon_{\beta}=78$, and $c_{s, \alpha} / c_{s, \beta}=\exp \left(\Psi_{D}\right)$.

The presence of an electrical potential difference $\Psi_{D}$ between the two phases leads to a negative contribution to the interfacial tension in a way that it is quadratic in $\Psi_{D}$, in line with the fact that the sign of the charge density should not matter. This quadratic dependence on $\Psi_{D}$ is also in line with the change in interfacial tension that results from the external application of an electrical potential difference $\Delta V$ as in electrowetting, $\Delta \gamma \propto(\Delta V)^{2}$, where the proportionality constant can be interpreted as an interfacial capacitance. An essential difference is that for the liquid-liquid interface investigated here, the potential difference results from the Donnan effect and could not be externally applied, as with oil-water interfaces [52], due to the low electrical resistance of the liquid-liquid interface.

Using Eq. (10), with $\Psi_{D}$ taken from the direct electrochemical measurements of the Donnan potential in Fig. 5(a), we can now subtract the electrostatic contribution to the interfacial tension. It turns out that for all three $\mathrm{pH}$ values considered here, the resulting intrinsic interfacial tensions $\gamma_{0}=\gamma-\Delta \gamma$ all collapse onto a single master curve when it is plotted as a function of the tie-line length [see Fig. 5(b)]. This result shows that the interfacial tension can be fully understood as the sum of two contributions: one term $\left(\gamma_{0}\right)$ that is related only to the relative position in the phase diagram as described by, for instance, the tie-line length, and a second (negative) term $(\Delta \gamma)$ related to the direct contribution to the interfacial tension due to the electrical potential difference across the interface. Although we have not shown it here, this conclusion is further corroborated by experiments performed under high salt conditions $(\Delta \gamma \ll \gamma)$ where it is observed that the interfacial tension no longer depends on polyelectrolyte charge and obeys the same master curve in Fig. 5(b).

Even though in the present experimental system the reduction of the interfacial tension due to charge can be up to $60 \%$ (see Fig. 3), this turns out to be insufficient to observe microdomain formation, as theoretically predicted in Refs. [34] and [36]. This shows the importance of the precise

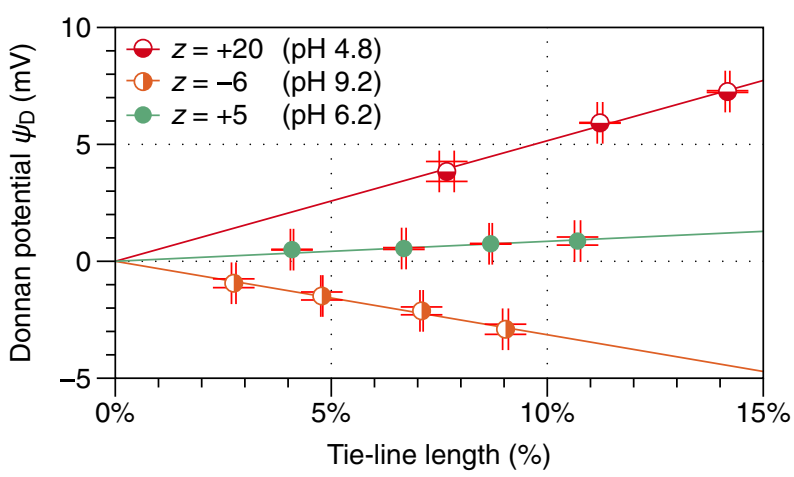

(a)

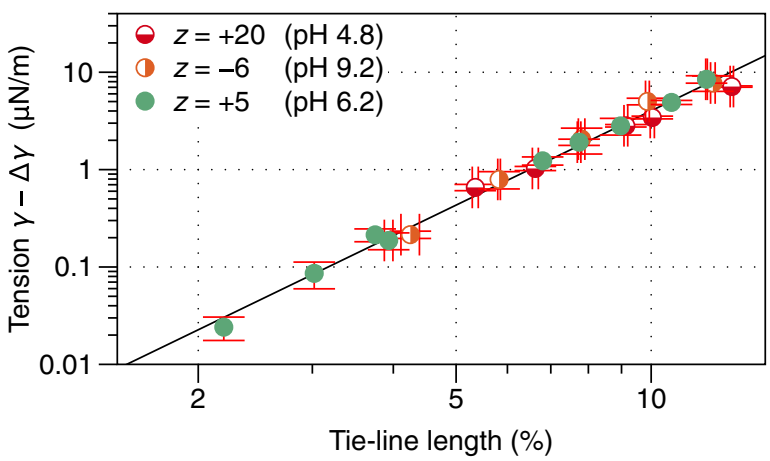

(b)

FIG. 5 (color online). Interfacial electrical potential difference $[40,41]$ and (b) interfacial tension compensated via Eq. (10) for the electrical potential difference of demixed aqueous solutions of dextran and nongelling fish gelatin. The potential and tension are plotted against the tie-line length (Fig. 2) at pH 6.2 with $5 \mathrm{mM}$ salt, pH 9.2 with $7 \mathrm{mM}$ salt, and $\mathrm{pH} 4.8$ with $9 \mathrm{mM}$ salt.

experimental conditions considered, i.e., poor-solvent conditions in Ref. [34] or melt conditions without added salt in Ref. [36]. In this respect, the result in Eq. (10) for the reduction in interfacial tension due to charge, helps us to speculate on the experimental conditions necessary to reduce the interfacial tension to zero, enabling the formation of microstructures. In general, such a reduction in the interfacial tension requires an increase in the Donnan potential which may be achieved either by decreasing the amount of added salt or by increasing the polyelectrolyte charge.

An interesting consequence of the reduction of the interfacial tension in these polymeric systems due to the presence of charge, is that the interfacial curvature energy becomes a relevant factor to describe, e.g., the possible occurrence and shapes of microstructures. It is expected that insight into the electric contribution to the interfacial curvature energy can be obtained by extending the Poisson-Boltzmann description presented here to curved surfaces as it was done for the curved charged solid-liquid interface [47].

In conclusion, electric charge is ubiquitous in soft matter but its role is often complicated and not well understood quantitatively. In the present Letter, we have shown that the interfacial tension of demixed aqueous solutions of a 
polyelectrolyte and a neutral polymer is reduced by the presence of the interfacial charge. The interfacial charge and interfacial electrical potential difference arise spontaneously due to the Donnan effect. By taking the effect of charge on the phase diagram into account, our precise and systematic measurements show that Poisson-Boltzmann theory quantitatively relates the decrease in tension to the measured potential difference.

The authors thank Paul van der Schoot for a helpful discussion. This work was supported by The Netherlands Organisation for Scientific Research (NWO).

*M.Vis@uu.nl

†B.H.Erne@uu.nl

[1] M. W. Beijerinck, Zentralbl. Bakteriol. Parasitenkd. Infektionskrankh. 22, 697 (1896).

[2] P.-Å. Albertsson, Nature (London) 182, 709 (1958).

[3] J. Ryden and P.-A. Albertsson, J. Colloid Interface Sci. 37, 219 (1971).

[4] D. Forciniti, C. K. Hall, and M. R. Kula, Fluid Phase Equilib. 61, 243 (1991).

[5] M. W. Edelman, E. van der Linden, and R. H. Tromp, Macromolecules 36, 7783 (2003).

[6] V. Y. Grinberg and V. B. Tolstoguzov, Carbohydr. Res. 25, 313 (1972).

[7] C. M. Durrani, D. A. Prystupa, A. M. Donald, and A. H. Clark, Macromolecules 26, 981 (1993).

[8] S. Kasapis, E. R. Morris, I. T. Norton, and M. J. Gidley, Carbohydr. Polym. 21, 249 (1993).

[9] V. Y. Grinberg and V. B. Tolstoguzov, Food Hydrocolloids 11, 145 (1997).

[10] V. Y. Grinberg, V. B. Tolstoguzov, and G. L. Slonimskii, Polym. Sci. USSR 12, 1810 (1970).

[11] M. W. Edelman, E. van der Linden, E. H. A. de Hoog, and R. H. Tromp, Biomacromolecules 2, 1148 (2001).

[12] H. G. Bungenberg de Jong and H. R. Kruyt, Proc. K. Ned. Akad. Wet. 32, 849 (1929).

[13] J. T. G. Overbeek and M. J. Voorn, J. Cell. Comp. Physiol. 49, 7 (1957).

[14] I. Michaeli, M. J. Voorn, and J. T. G. Overbeek, J. Polym. Sci. 23, 443 (1957).

[15] I. Capron, S. Costeux, and M. Djabourov, Rheol. Acta 40, 441 (2001).

[16] V. Tolstoguzov, Food Hydrocolloids 17, 1 (2003).

[17] A. T. Poortinga, Langmuir 24, 1644 (2008).

[18] H. Firoozmand, B. S. Murray, and E. Dickinson, Langmuir 25, 1300 (2009).

[19] I. Ziemecka, V. van Steijn, G. J. M. Koper, M. T. Kreutzer, and J. H. van Esch, Soft Matter 7, 9878 (2011).

[20] G. Balakrishnan, T. Nicolai, L. Benyahia, and D. Durand, Langmuir 28, 5921 (2012).

[21] B. T. Nguyen, T. Nicolai, and L. Benyahia, Langmuir 29, 10658 (2013).

[22] B. T. Nguyen, W. Wang, B. R. Saunders, L. Benyahia, and T. Nicolai, Langmuir 31, 3605 (2015).

[23] L. De Ruiter and H. G. Bungenberg de Jong, Proc. K. Ned. Akad. Wet. 50, 836 (1947).
[24] V. G. Langhammer and L. Nestler, Makromol. Chem. 88, 179 (1965).

[25] D. Forciniti, C. K. Hall, and M. R. Kula, J. Biotechnol. 16, 279 (1990).

[26] P. Ding, B. Wolf, W. J. Frith, A. H. Clark, I. T. Norton, and A. W. Pacek, J. Colloid Interface Sci. 253, 367 (2002).

[27] Y. A. Antonov, P. Van Puyvelde, and P. Moldenaers, International Journal of Biological Macromolecules 34, 29 (2004).

[28] M. Simeone, A. Alfani, and S. Guido, Food Hydrocolloids 18, 463 (2004).

[29] E. Scholten, R. Tuinier, R. H. Tromp, and H. N. W. Lekkerkerker, Langmuir 18, 2234 (2002).

[30] E. Scholten, J. E. Visser, L. M. C. Sagis, and E. van der Linden, Langmuir 20, 2292 (2004).

[31] R. H. Tromp and E. M. Blokhuis, Macromolecules 46, 3639 (2013).

[32] E. Spruijt, J. Sprakel, M. Stuart, and J. van der Gucht, Soft Matter 6, 172 (2010).

[33] J. Qin, D. Priftis, R. Farina, S. L. Perry, L. Leon, J. Whitmer, K. Hoffmann, M. Tirrell, and J. J. de Pablo, ACS Macro Lett. 3, 565 (2014).

[34] V. Y. Borue and I. Y. Erukhimovich, Macromolecules 21, 3240 (1988).

[35] J. F. Joanny and L. Leibler, J. Phys. (France) 51, 545 (1990).

[36] I. A. Nyrkova, A. R. Khokhlov, and M. Doi, Macromolecules 27, 4220 (1994).

[37] F. G. Donnan, Z. Elektrochem. Angew. Phys. Chem. 17, 572 (1911).

[38] F. G. Donnan, Chem. Rev. 1, 73 (1924).

[39] A. P. Philipse and A. Vrij, J. Phys. Condens. Matter 23, 194106 (2011).

[40] M. Vis, V.F. D. Peters, R. H. Tromp, and B. H. Erné, Langmuir 30, 5755 (2014).

[41] M. Vis, V.F. D. Peters, B. H. Erné, and R. H. Tromp, Macromolecules 48, 2819 (2015).

[42] R. H. Tromp, M. Vis, B. H. Erné, and E. M. Blokhuis, J. Phys. Condens. Matter 26, 464101 (2014).

[43] D. G. A. L. Aarts, J. van der Wiel, and H. N. W. Lekkerkerker, J. Phys. Condens. Matter 15, S245 (2003).

[44] M. B. Perrau, I. Iliopoulos, and R. Audebert, Polymer 30, 2112 (1989).

[45] K. Bergfeldt and L. Piculell, J. Phys. Chem. 100, 5935 (1996).

[46] B. Widom and J.S. Rowlinson, Molecular Theory of Capillarity (Oxford University Press, Oxford, 1984).

[47] H. N. W. Lekkerkerker, Physica (Amsterdam) 167A, 384 (1990).

[48] J. Frenkel, Kinetic Theory of Liquids (Dover Publications, New York, 1955), Chap. VI.

[49] E. J. W. Verwey and J. T. G. Overbeek, Theory of the Stability of Lyophobic Colloids (Dover Publications, New York, 1999), Chaps. II and III.

[50] E. Ruckenstein and R. Krishnan, J. Colloid Interface Sci. 76, 201 (1980).

[51] H. Ohshima and S. Ohki, J. Colloid Interface Sci. 103, 85 (1985).

[52] A. G. Volkov, D. W. Deamer, D. L. Tanelian, and V. S. Markin, Prog. Surf. Sci. 53, 1 (1996). 